\title{
Measuring community severance for transport policy and project appraisal
}

\author{
P. R. Anciaes \\ London School of Economics and Political Science, UK
}

\begin{abstract}
The construction of transport infrastructure often leads to the separation of neighbourhoods, with effects on local mobility and community well-being. While these effects are usually included in transport appraisal, in practice, choices over alternative projects or route alignments tend to be subject to ad-hoc political decisions based on qualitative assessments. The development of quantitative indicators of severance can bring more transparency to these decisions, contributing to their socio-political feasibility. In addition, there is little evidence on the effects of past decisions on present degrees of severance in different parts of an urban area. This paper proposes indicators for measuring community severance based on the lost population-interaction potential and considering alternative definitions of restrictions to pedestrian mobility. These include the barrier effect of motorways and railways, the dynamic severance of traffic on busy roads, and the cumulative effect of transport infrastructure located near other pedestrian-unfriendly environments. The indicators are used in two applications in the Lisbon Metropolitan Area, evaluating the severance effects of a recently implemented road project and the overall effects of the transport infrastructure projects implemented during a 10-year period.
\end{abstract}

Keywords: community severance, local accessibility, pedestrian mobility.

\section{Introduction}

Community severance is one of the most significant local impacts of the urban transport system. The construction of roads and railways often carries changes in the street network, separating communities and contributing the isolation of some neighbourhoods. These changes decrease local accessibility and increase walking times to reach nearby places and may have psychological effects on 
local residents, with implications on the number of inter-neighbourhood trips and community interactions [1].

Community severance effects are often included in transport project appraisal, and transport departments in several countries have produced guidelines for the assessment of these effects. However, these guidelines tend to propose indicators that are not directly related to the location of transport infrastructure but rather to the future traffic on that infrastructure, such as traffic levels, speeds and noise. While these indicators are suitable for assessing environmental and safety concerns, they may not evaluate correctly the effect of transport infrastructure on local mobility. On the other hand, the few existing measures based on the effects of infrastructure tend to be statistics of the population living within a certain distance of the infrastructure or of the effect on the accessibility to local facilities [2]. These measures do not fully capture the barrier effect as they do not consider the patterns of the street network and the intra-urban variations in accessibility needs and in the density of urban facilities.

Regardless of the suitability of the indicators used in project appraisal studies, the design of road or railway alignments that separate two communities tends to be a political question and is generally subject to much controversy. The development of quantitative indicators can therefore benefit policy-makers by supporting the assessment of the social interest and socio-political feasibility of transport projects. The indicators can also contribute to the inclusion of local populations in the public choice process, as they represent practical tools that can be used by these populations to evaluate and illustrate the potential effects of transport projects at the neighbourhood level.

At a more strategic level, there is also a scarcity of methods that allow for global assessments of the severance impacts of past transport policies. In recent years, academics have shown growing interest in mapping indicators of local mobility and analysing their intra-urban variations. However, most of the research has focused on the local availability of pedestrian destinations [3, 4]. There is less evidence on the spatial variability of the quality of local mobility and on the extent to which this mobility is restricted by transport infrastructure. This paper contributes to this subject by studying the spatial distribution and evolution of levels of community severance using objective measures based on models of the street network and covering a metropolitan area.

The definition of an operational measure of severance depends on the concepts of "community" and of what separates communities [5]. The novelty of this paper is to measure severance as the effect of infrastructure in the interaction potential between the populations living in different neighbourhoods. Severance is defined as the restriction on the possibility (or loss of amenity value) of walking to access nearby locations in order to meet people. In the following section, three alternative indicators are proposed, based on different definitions of barriers to pedestrian mobility. In Sections 3 and 4, those indicators are applied to the study of the effects of a large infrastructure project and the overall effects of the projects implemented in the Lisbon Metropolitan Area during a 10year period. A final section concludes the paper and proposes future directions for research. 


\section{Methods}

The community severance indicators are defined at the level of the census enumeration district. To take into account the size and heterogeneity of enumeration districts in less urbanized areas, the indicators are estimated for all contiguous residential areas inside each district and then average to the district level. The modelling of the transport network and pedestrian routes uses a Geographic Information System (GIS) and is based on the framework represented in Figure 1. Pedestrians walk from a representative point of the district (O) to nearby destinations (D). The set of all possible destinations in the metropolitan area is obtained by the largest possible sample of all inhabited points in the metropolitan area such that points are at least $400 \mathrm{~m}$ apart and the probability that each point is included in the sample is proportional to its estimated population density, calculated over a population surface model. The specific set of destinations for a given district includes all points from this sample located within $800 \mathrm{~m}$ straight-line distance of the district's representative point. The restrictions on the spacing of destinations and on the set of destinations accessible at each district ensure that each point $\mathrm{O}$ has between 4 and 12 possible destinations.

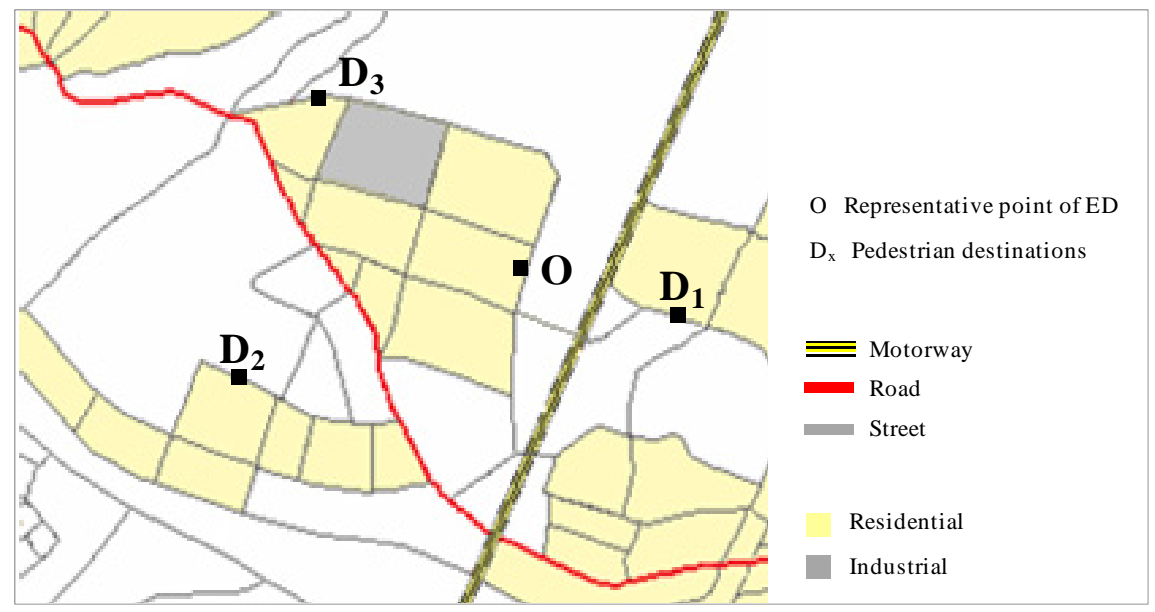

Figure 1: $\quad$ Framework for the measurement of community severance.

The population of the metropolitan area is then assigned to the nearest point in the set of all possible destination points. In each district, the attractiveness of each destination is equal to its assigned population. It is therefore assumed that the need to access nearby destinations on foot is directly explained by population interaction. However, this assumption also accounts for other trip purposes, as population density tends to be correlated with other local attractiveness factors such as the location of shops, urban facilities or local jobs. The attractiveness index is corrected by a factor equal to 0.5 for destinations within 500 and $800 \mathrm{~m}$ 
straight-line distance. The threshold values of 500 and $800 \mathrm{~m}$ are based on the indicative distances of the "Streets for Life" urban design concept developed at Oxford Brookes University, which suggests two levels of reasonable walking distances [6].

Three alternative indicators are considered, based on alternative definitions of what constitutes a barrier to the mobility of pedestrians between different neighbourhoods.

The first indicator considers barriers posed by transport links where pedestrian crossing is only possible at a limited number of locations, using footbridges and overpasses. It is assumed that infrastructure is a barrier to the movement of pedestrians even when these facilities are present, due to the intimidation effect of the infrastructure and traffic at high speeds. The set of relevant links include restricted-access transport infrastructure such as railways and motorways, dual carriageways and respective access roads.

The second indicator includes a wider set of transport links, independently of their restrictions to pedestrian traffic. The links added allow pedestrian crossing at a large number of locations (such as pedestrian crossings) but there is a severance effect due to the road characteristics or large traffic levels, noise and pedestrian accident risk. The indicator then captures the "dynamic barrier" posed by road traffic to the mobility of local populations [7]. The set of relevant links includes roads with multiple lanes or large traffic levels. These are identified respectively by visual inspection of satellite images and orthophoto maps and by mapping road traffic data.

A third indicator adds barriers posed by non-transport infrastructure and land uses. The indicator intends to measure the cumulative effects of transport and non-transport barriers to local mobility. Non-transport barriers are defined as infrastructure or areas that, due to their morphological or aesthetical characteristics, have a noticeable and negative effect on the propensity to walk or on the amenity-value of walking. These barriers are identified in land use maps and gazetteers and include industrial estates, ports, storage areas, mining areas/quarries, military installations, airports, aerodromes, water reservoirs, electricity transformation poles and waste management centres.

Community severance in a given enumeration district is then defined as the proportion of the population-interaction potential in that district's set of destinations that cannot be reached on the street network unless crossing a barrier. Pedestrian routes are determined using a network analyst tool within the GIS. In the example in Figure 1, the calculation of the indicator considering restricted-access transport barriers labels destination $\mathrm{D}_{1}$ as "unreachable" for the population living in point $\mathrm{O}$. The indicator for all transport barriers adds $\mathrm{D}_{2}$ to the list of unreachable destinations, while the indicator for transport and nontransport barriers also adds $\mathrm{D}_{3}$ to that list.

\section{Assessment of individual projects}

In this section, the methodology described above is applied to the assessment of the severance effects of a large road infrastructure project in Lisbon. This project 
is the final section of the Lisbon Inner Ring Road (“CRIL”), a $21 \mathrm{~km}$ dual carriageway road enclosing the Lisbon municipality. While most of the sections in the CRIL opened between 1993 and 1998, the construction of the final $3 \mathrm{~km}$ section was delayed for almost 20 years, finally opening in 2011. The reason of the delay lies in the technical, economic, social, political and legal factors associated with the location of this section, as it crosses densely populated neighbourhoods. Despite the construction of tunnels, in most areas the motorway is a severe barrier to the mobility of the local populations (Figure 1).

Figure 3 maps the increase of community severance in the districts surrounding the road. As the CRIL is a restricted-access road, the increase is the same for all three indicators. The map shows the differences in the sizes of the

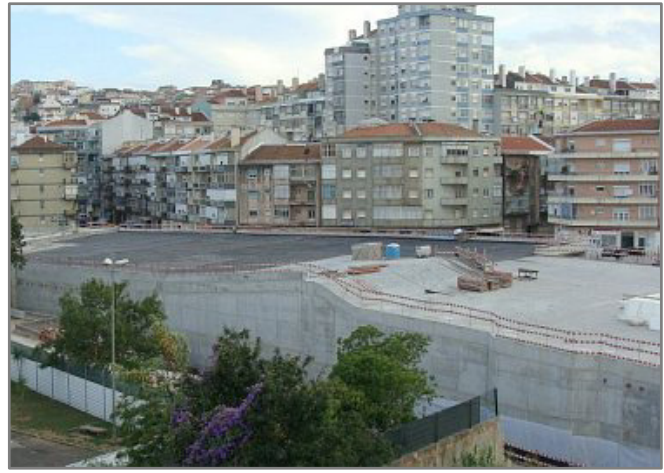

Figure 2: $\quad$ The CRIL during construction (Source: www.cril-segura.com).

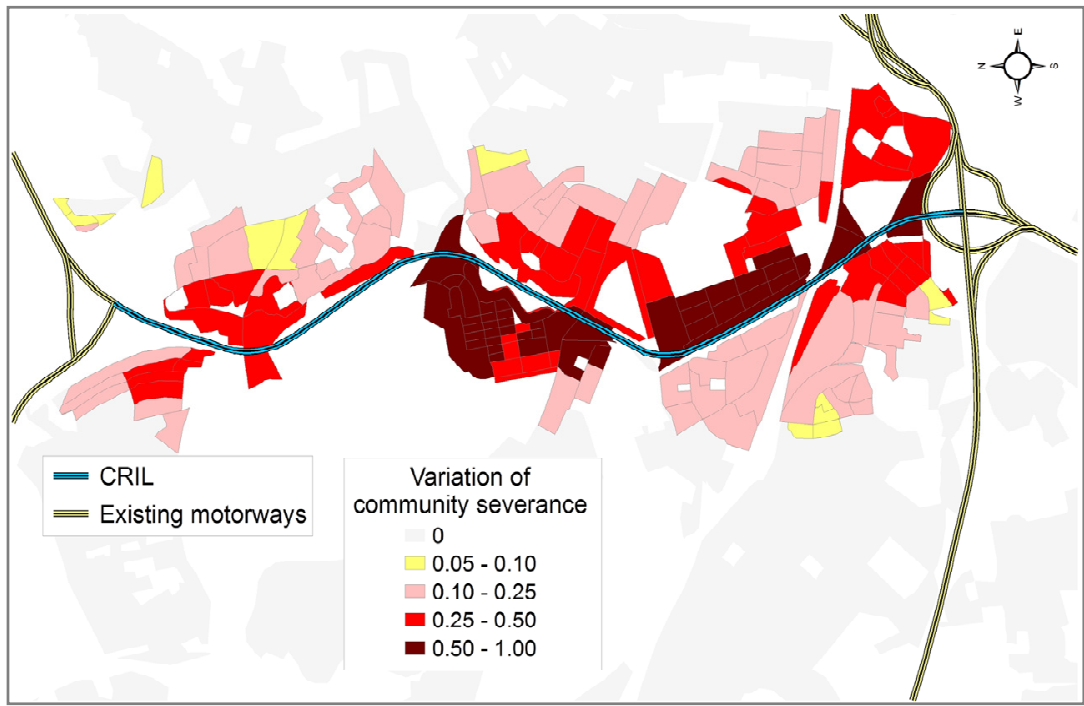

Figure 3: $\quad$ Severance effects of the final section of Lisbon inner ring road. 
effect in districts located at similar distances from the road, illustrating the relevance of considering the size of population living on the opposite side of the road when assessing community severance. The effect is especially acute in areas for which that population represents a high proportion of the surrounding population, such as in areas already surrounded by other transport infrastructure or by non-residential land uses.

Table 1 gives statistics of the severance effect and characterizes the affected neighbourhoods in terms of initial (pre-project) severance indicators and demographic variables, comparing them with the whole population in the two affected municipalities (Lisbon and Amadora).

Table 1: $\quad$ Statistics of severance effects.

\begin{tabular}{|ccc|}
\hline & $\begin{array}{c}\text { Affected } \\
\text { neighbourhoods }\end{array}$ & Municipalities \\
\hline Severance effect & & \\
Population affected & 42305 & \\
Lost population-interaction potential (mil) & 135.6 & \\
Average effect & $27.7 \%$ & \\
Maximum effect & $73.6 \%$ & $10 \%$ \\
Initial severance level (weighted avg.) & & $24.3 \%$ \\
Transport (restricted-access) & $13.4 \%$ & $32.4 \%$ \\
All transport & $28.3 \%$ & \\
Transport and non-transport & $32.1 \%$ & $11.5 \%$ \\
Demographics (weighted avg.) & & $21.5 \%$ \\
Children & $13.5 \%$ & $28.5 \%$ \\
Elderly & $17.6 \%$ & $7.6 \%$ \\
Low-qualified & $27.5 \%$ & $4.8 \%$ \\
Unemployed & $7.0 \%$ & $6.1 \%$ \\
Ethnic minorities & &
\end{tabular}

The project has an effect on the potential walking routes of over 42000 people, representing more than 135 million potential population interactions. This value is obtained by summing up the product of the population at the origins and destinations of affected pedestrian routes. In average, there is a loss of $27.7 \%$ of the population interaction potential in each district, while in the most affected district, $73.6 \%$ of the potential is lost.

The second part of the table gives the averages of the initial levels of the indicator, weighted by population. The project affects an area which already has a higher severance index than the other neighbourhoods in the two municipalities, when considering either restricted-access transport infrastructure or the wider set of transport barriers. The project then contributes to the deterioration of pedestrian mobility in an area where there this type of mobility already faces limitations, a factor which must be taken into consideration when judging the social equity aspects of the project. 
The third part of the table characterizes the affected area in terms of the proportions of groups usually considered as especially vulnerable to community severance and losses of pedestrian mobility, such as children, the elderly, lowqualified and unemployed individuals, and ethnic minorities. The values are averages of the affected districts, weighted by population and size of the effect. The averages in the two municipalities are weighted by the population in each district. The affected neighbourhoods have higher proportions of children and racial minorities but lower proportions of elderly, low-qualified and unemployed individuals. These results suggest that the areas affected by severance may have a specific socio-economic profile, which must be taken into account in the design and evaluation of the project, considering issues such as monetary compensation, application of mitigation policies, and prevention of possible impacts on public health and social cohesion.

\section{Assessment of broad transport strategies}

This section generalizes the previous one by applying the proposed severance indicators to the study of the effects of the set of all transport projects implemented within a period of time. The indicators are estimated for all enumeration districts of the Metropolitan Area of Lisbon at the moment of the two last published census (1991 and 2001), considering the existing transport network in those years. The period in question was characterized by major changes in the metropolitan motorway and arterial road network, including the outer and most of the inner Lisbon ring roads and a series of radial motorways linking the outskirts of Lisbon with the different corridors in the metropolitan area and with the rest of the country. Many national and municipal roads have also been redesigned or upgraded, often by adding extra lanes. The only new railway line opened in this period was the rail connection between the north and the south banks of the Tejo River.

The spatial distribution of the indicator incorporating all transport barriers is represented in Figure 4. The map shows the intensification of the severance effect in the Lisbon municipality and an extension of the effect in other municipalities. While in 1991 the effect roughly follows a radial pattern originating from the Lisbon CBD, in 2001 the effect tends to be more widespread. The severance effect is noticeable not only in narrow commuting corridors, such as the Northwest and Northeast commuting corridors to Lisbon, but also in some isolated semi-rural communities. In both cases, this is explained by the fact that the lost population interaction potential in both set of areas represents a relatively high proportion of the total potential due to their separation from other neighbourhoods. This separation is transport-based in the first case and is geographical in the second case.

Figure 5 gives the population affected by each severance level, by type of barriers, region and year. The values are expressed as percentages of the population in each region and year. The analysis is segmented by region due to the heterogeneity of the metropolitan area in terms of urbanization levels and the divide between the north and south banks. The delimitation of the commuting 

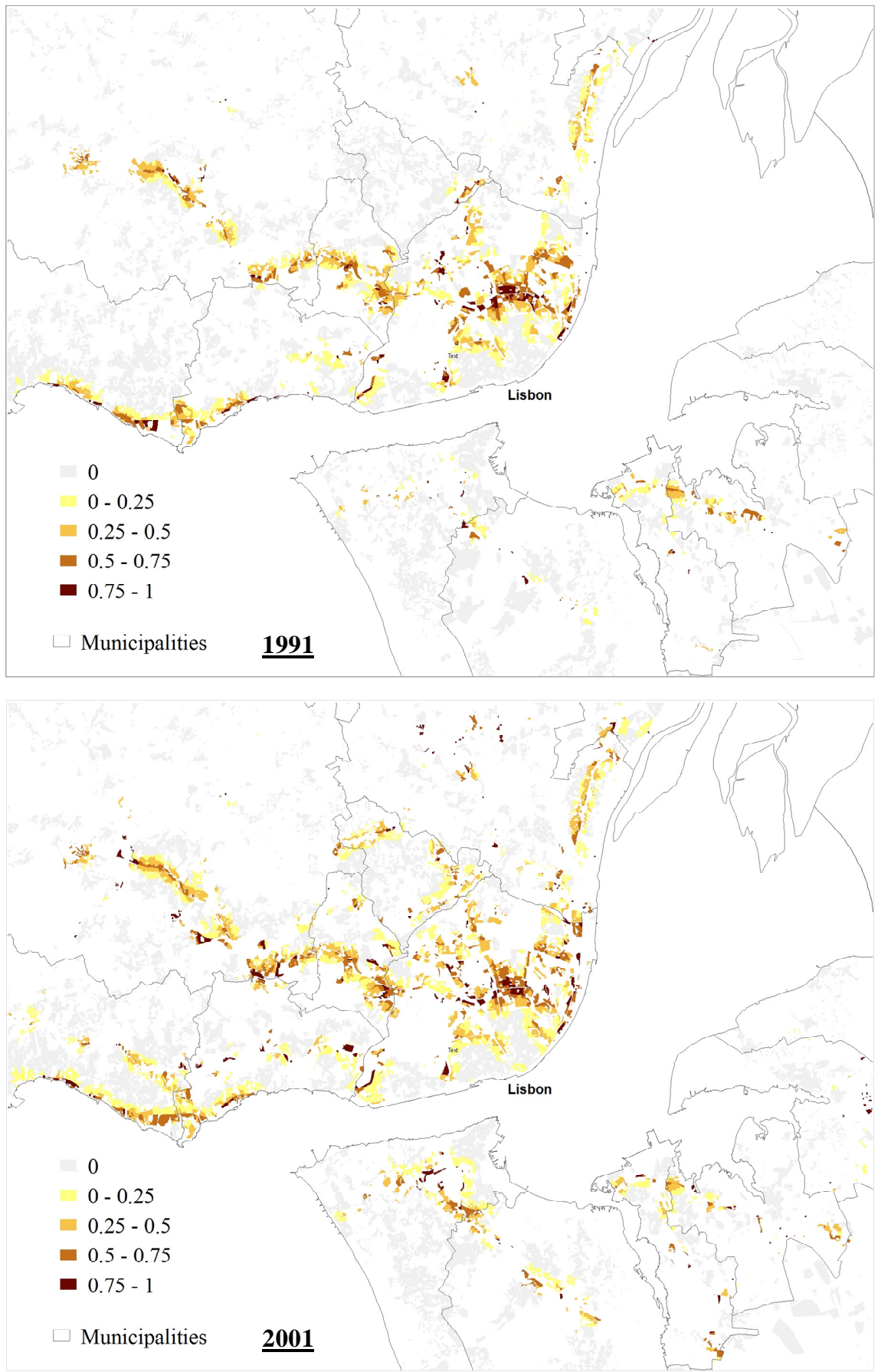

Figure 4: $\quad$ Severance in the metropolitan area of Lisbon (all transport). 

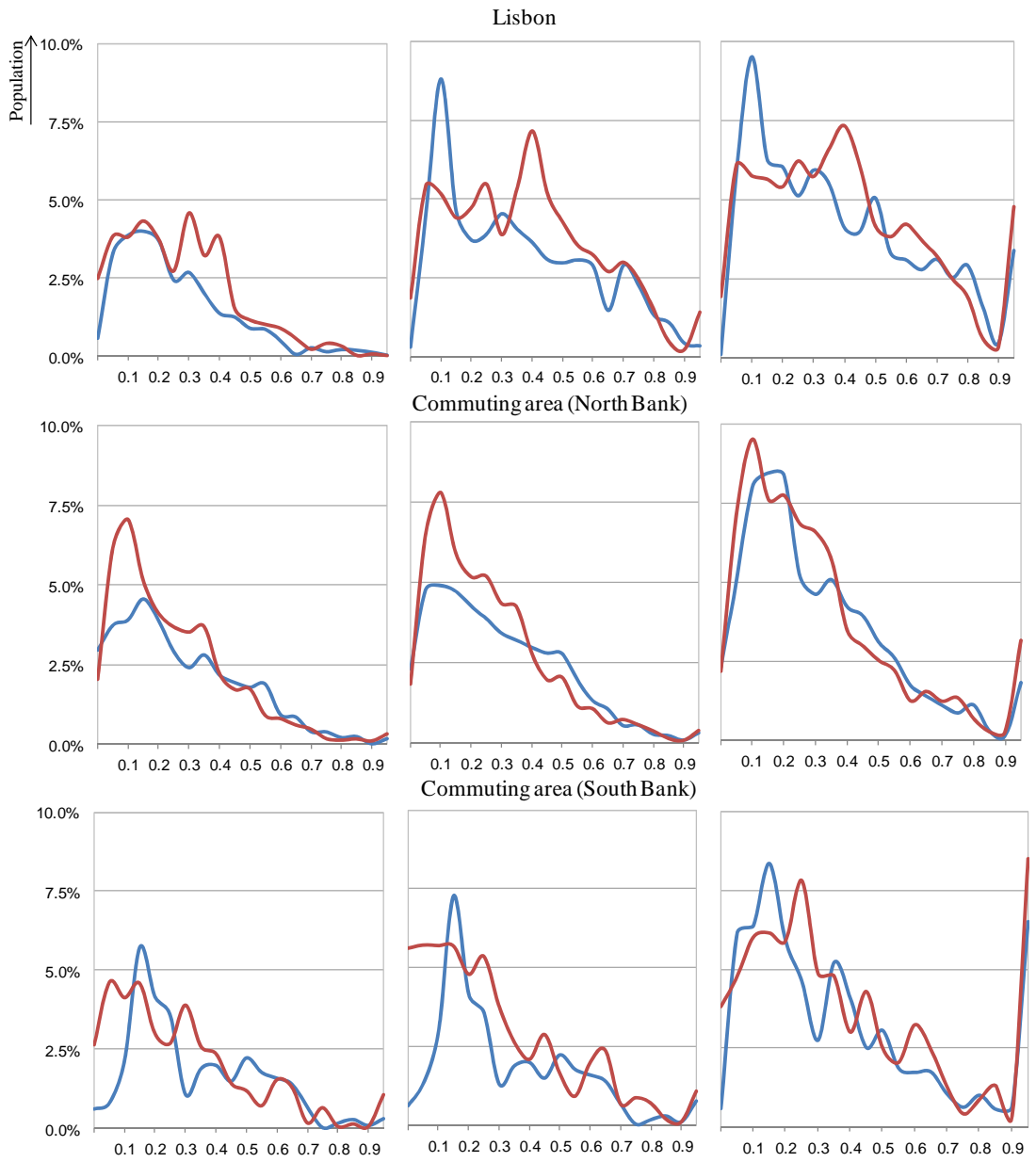

$\begin{array}{lllllllll}0.1 & 0.2 & 0.3 & 0.4 & 0.5 & 0.6 & 0.7 & 0.8 & 0.9\end{array}$
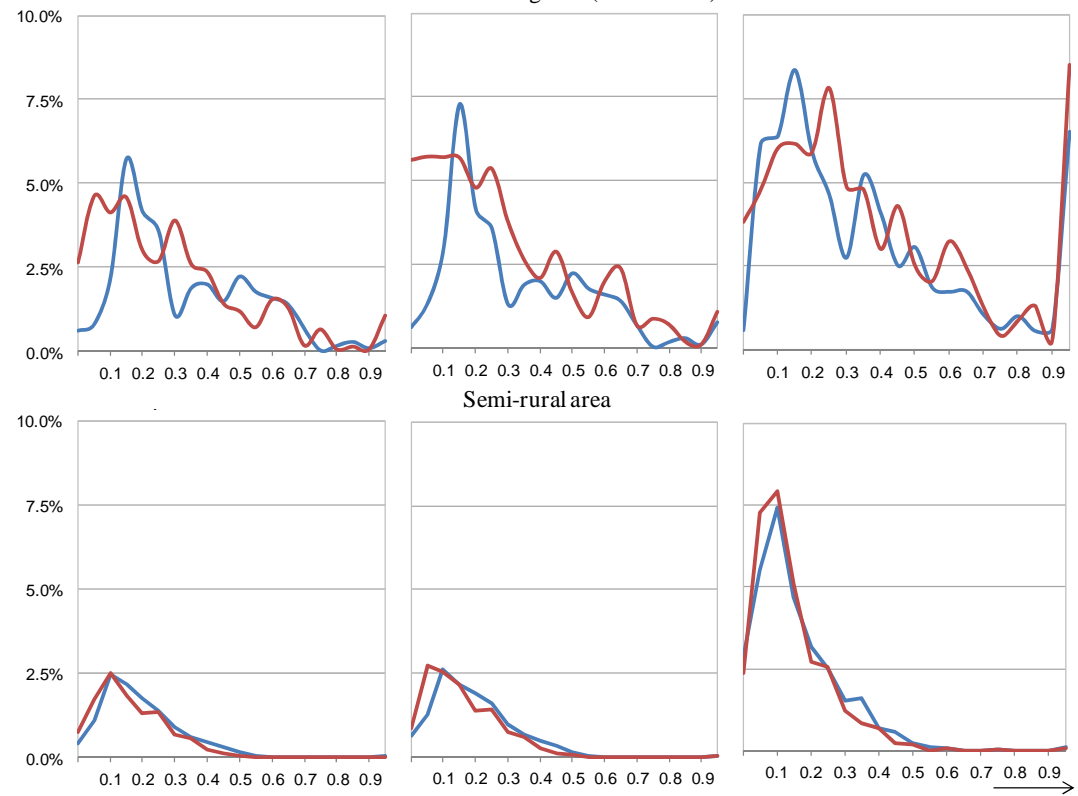

$\begin{array}{llllllll}0.2 & 0.3 & 0.4 & 0.5 & 0.6 & 0.7 & 0.8 & 0.9\end{array}$ Semi-rural area

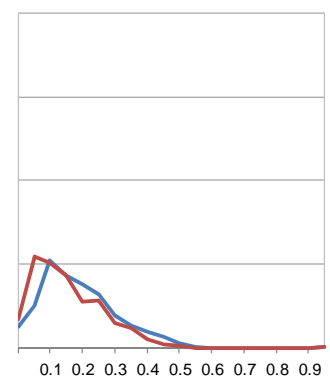

Transport (restricted access)
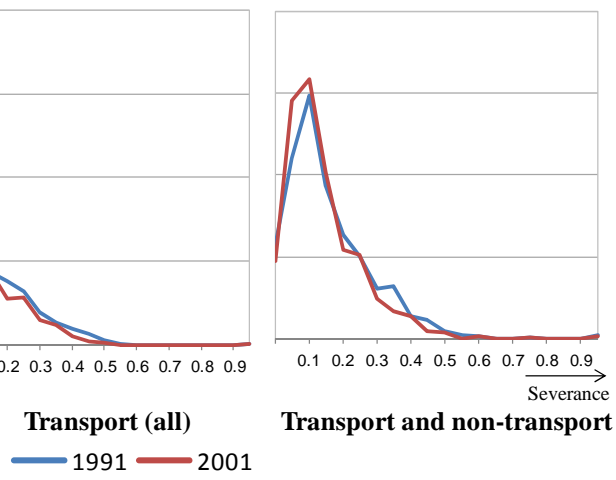

Transport and non-transport

Figure 5: Population affected by each level of severance, by type of barriers, year and region. 
area is based on a previous analysis to levels of private transport accessibility to Lisbon. The remaining districts are semi-rural and are located in the peripheral parts of the metropolitan area.

The population in Lisbon municipality is relatively spared from the severance caused by restricted-access transport infrastructure, comparing with the suburban areas, as only a couple of motorways cross the municipality. However, Lisbon has a large density of busy arterial roads in Lisbon, leading to a high severance effect when using the indicator incorporating all transport barriers. As the presence of large industrial sites or and similar equipment in Lisbon is rare, adding non-transport barriers does not change the severance profile of the districts. There was a marked aggravation of the problem from 1991 to 2001, with a larger proportion of population in the ]0.25-0.5] interval of the "restricted-access only" indicator and a shift of population in the ]0-0.25] interval to the ]0.25-0.5] interval in the indicator for all barriers.

The distribution of the frequencies of the severance effect in the commuting areas roughly follows a Poisson distribution with small parameter, most noticeably in the north bank. In this area, there was an increase of the frequencies of the lower levels of severance from 1991 to 2001. In the south bank, the distribution became slightly less variable. In both areas, and contrary to the case of Lisbon, the differences in the profile of the two indicators based on transport barriers are small, while the difference between the indicator based on all transport barriers and the indicator adding non-transport barriers are considerable, especially in the south bank. In this area, there is also a high percentage of populations with the most extreme levels of severance $(>0.9)$ when considering the last indicator.

In the semi-rural areas, the dimension of the problem is substantially lower than in other areas, when looking at the transport-related indicators. Furthermore, there are little differences between the two indicators and between the situation in 1991 and 2001. However, the addition of non-transport barriers increases the proportion of affected population, although only in the lower intervals of the effect.

\section{Conclusions and further work}

This paper assessed the severance effects of transport in an urban area. The research contributes to the literature by designing indicators based on the effects on pedestrian mobility, considering alternative definitions of barriers to mobility and using GIS to measure the effects on pedestrian routes to local destinations. The indicators obtained can be applied not only in project appraisal but also on the overall evaluation of a set of projects implemented in a given period, adding to the growing literature studying intra-urban variations in pedestrian mobility and accessibility.

Given the subjectivity inherent to the definition of the problem, there is a need for further research, in order to define objective measures and analyse comparatively the different dimensions or alternative definitions of community severance [8]. In that sense, by focusing on population interaction potential, this 
paper complements existing studies dealing with the effects of community severance on accessibility to local facilities. There are also several nuances in the definition of barriers to mobility which have not been fully explored in this paper. In particular, there is scope for the inclusion of mitigation factors in the assessment of the effect of a barrier on mobility in the cases of sections of transport infrastructure where pedestrian crossing is allowed through footbridges, underpasses or pedestrian crossings.

It is also important to complement the study of potential measures of severance with the results of surveys to the actual effects of the infrastructure on local communities, such as suppressed pedestrian trips, time losses, and physical and mental health effects or social problems associated with reduced pedestrian mobility.

Finally, it should be noted that the assessment of the size of severance effects represents the first step towards their inclusion in transport appraisal. The monetisation of the effects is a necessary second step for their integration in economic-based evaluation methods such as cost-benefit analyses. However, several additional assumptions must be made to derive monetary values of severance effects, increasing the subjectivity of their assessment [9]. Ultimately, severance must be evaluated in terms of trade-offs with the benefits of the infrastructure and with the other local and non-local economic, social and environmental costs. Social equity aspects are also crucial to this assessment, especially when community severance reduces the mobility of vulnerable groups in society.

\section{Acknowledgements}

The author thanks Giles Atkinson, Steve Gibbons, Andrew Lovett and Helena Titheridge for their helpful comments on early versions of this paper. This work was partly supported by the Portuguese Foundation for Science and Technology.

\section{References}

[1] Bradbury, A., Tomlinson, P. and Millington, A. Understanding the evolution of community severance and its consequences on mobility and social cohesion over the past century. Proceedings of the European Transport Conference 2007, 17-19 October 2007, Leeuwenhorst, The Netherlands, http://www.etcproceedings.org/paper/download/2750, 2007.

[2] Clark, J. M., Hutton, B. J., Burnett, N., Hathway, A. and Harrison, A. The Appraisal of Community Severance. Report for the Transport and Road Research Laboratory. Contractor report 135. Transport and Road Research Laboratory, UK Department of Transport: Crowthorne, 1991.

[3] Cerin, E., Leslie, E., Du Toit, L., Owen, N. and Frank, L. D. Destinations that matter: Associations with walking for transport. Health and Place 13 (3), pp. 713-724, 2007. 
[4] Witten, K., Pearce, J. and Day, P. Neighbourhood Destination Accessibility Index: a GIS tool for measuring infrastructure support for neighbourhood physical activity. Environment and Planning A 43 (1), pp. 205-223, 2011.

[5] Millington, J. E. and Tomlinson, P. Understanding community severance Part 1: Views of Practitioners and Communities. Report for the UK Department of Transport, http://www.uktrc.ac.uk/documents/dft/2005/ Understanding Community Sev1.pdf, 2005

[6] Burton, E. and Mitchell, L. Inclusive Urban Design: Streets For Life. Architectural Press: Oxford, 2006.

[7] Guo, X., Black, J. and Dunne, M. Crossing pedestrians and dynamic severance on urban main roads. Road \& Transport Research Journal 10 (3), pp. 84-98, 2001.

[8] Mindell, J. S. and Karlsen, S. Community Severance and Health: What do we actually know? Journal of Urban Health 89 (2), pp. 232-246, 2012

[9] Tomlinson, P. and James, E. Understanding community severance Part 2: Monetisation of Severance Impacts. Report for the UK Department of Transport, http://www.uktrc.ac.uk/documents/dft/2005/Understanding Community_Sev2.pdf, 2005. 